\title{
Microvascular Obstruction in Acute Myocardial Infarction
}

\author{
Ioana Dregoesc ${ }^{1,2}$, Adrian Iancu ${ }^{1,2}$, Simona Manole ${ }^{3}$, Şerban Bălănescu4 \\ ${ }^{1}$ Department of Cardiology, "Iuliu Haţieganu" University of Medicine and Pharmacy, Cluj-Napoca, Romania \\ 2 Department of Cardiology, "Niculae Stãncioiu" Heart Institute, Cluj-Napoca, Romania \\ ${ }^{3}$ Department of Radiology, "Iuliu Haţieganu" University of Medicine and Pharmacy, Cluj-Napoca, Romania \\ 4 "Carol Davila" University of Medicine and Pharmacy, Bucharest, Romania
}

\section{ABSTRACT}

Introduction: The no-reflow phenomenon has been described in $20-40 \%$ of patients with acute ST-segment elevation myocardial infarction, despite restoration of TIMI 3 myocardial flow. It is associated with adverse left ventricular remodeling and an unfavorable long-term prognosis. Case presentation: A 45-year-old gentleman was admitted one hour after the onset of an acute anterior ST-segment elevation myocardial infarction. Emergency coronary angiography was performed, and a severe stenosis of the left anterior descending artery was identified. The lesion was crossed with a pressure-wire, and a drug-eluting stent was directly implanted, with restoration of TIMI 3 epicardial flow. Predilatation was not performed. Coronary wedge pressure was measured during stent deployment. The mean pressure value was $27 \mathrm{mmHg}$. However, a tall systolic wave was identified in the morphology of the pressure curve. Myocardial blush grade and ST-segment resolution were concordant with early microvascular obstruction. Similarly, at transthoracic Doppler echocardiography, the flow in the left anterior descending artery revealed the same pattern. An apical left ventricular aneurysm was echocardiographically detected. The MRI described extensive interstitial edema that affected the anterior, septal, and apical regions of the left ventricle. Areas of intramyocardial hemorrhage and microvascular obstruction were also detected. According to recent literature data, the morphology of the coronary wedge pressure wave suggested at least the presence of pre-procedural distal embolization. Conclusions: In the setting of acute myocardial infarction, the integrity of coronary microvasculature is an important issue. The distal coronary pressure wave pattern before primary percutaneous revascularization can be a deciding factor for an early therapeutic approach.

Keywords: ST-segment elevation myocardial infarction, coronary wedge pressure, microvascular obstruction, inflammation, interstitial edema

\section{ARTICLE HISTORY}

Received: December 10, 2017

Accepted: December 17, 2017

\section{CORRESPONDENCE}

Adrian Iancu

Calea Moţilor 19-21

400001 Cluj-Napoca, Romania

Tel: +40 744751027

E-mail: adrian_iancu@hotmail.com 


\section{INTRODUCTION}

The no-reflow phenomenon is associated with microvascular dysfunction. It has been described in $20-40 \%$ of patients with acute ST-segment elevation myocardial infarction (STEMI), despite restoration of TIMI 3 myocardial flow. ${ }^{1}$

It is associated with a fivefold increase in the risk of myocardial infarction and a fourfold increase in the risk of death. ${ }^{2}$ No-reflow has also been associated with an increased risk of left ventricular systolic dysfunction, reduced left ventricular ejection fraction, left ventricular remodeling, malignant ventricular arrhythmias, heart failure, and cardiac rupture. ${ }^{3,4}$

None of the prophylactic and therapeutic approaches currently available are effective to prevent this phenomenon. ${ }^{5-8}$

\section{CASE PRESENTATION}

A 45-year-old gentleman, with a 30 pack-year smoking history and recurrent episodes of exertional angina during the last six months, was admitted to the Emergency Department one hour after the onset of an acute anterior STEMI. At the time of admission, the patient was in Killip class II. The ECG recording showed ST-segment elevation in the precordial leads (Figure 1). Double antiplatelet therapy with $250 \mathrm{mg}$ aspirin and $180 \mathrm{mg}$ ticagrelor was administered, together with $80 \mathrm{mg}$ atorvastatin.

Emergency coronary angiography was performed through $6 \mathrm{~F}$ right radial access, and a proximal non-collateralized left anterior descending (LAD) artery sub-occlusion was identified. TIMI 2 flow was recorded in the distal LAD. The patient was anticoagulated with $100 \mathrm{UI} / \mathrm{kg}$ of unfractionated heparin.
A 6F extra back-up guiding catheter (Launcher, Medtronic, Minneapolis, MN) was selected, and the lesion was crossed with a 0.014" pressure guidewire (Verrata Pressure Guide Wire, Volcano Corporation, San Diego, CA). A 3.0/23mm everolimus-eluting stent (Promus Element Plus, Boston Scientific, Marlborough, MA) was implanted in the proximal LAD, and TIMI 3 flow was obtained. However, myocardial blush grade (MBG) was zero (Figure 2). During stent deployment, the distal coronary pressure was measured through the tip of the pressure-wire. The recorded mean value was $27 \mathrm{mmHg}$. A tall systolic wave was identified in the pattern of the pressure curve (Figure 3).

The ECG recording that was performed after the procedure showed the persistence of ST-segment elevation.

The first high-sensitivity troponin $\mathrm{T}$ value was in the normal range. However, 12 hours after the procedure, it reached a peak value of $3.78 \mathrm{ng} / \mathrm{ml}$, which suggested a large area of necrosis. Left ventricular ejection fraction was $35 \%$, as evaluated by transthoracic echocardiography. An apical left ventricular aneurysm was identified. LAD flow was analyzed by Doppler echocardiography. A diastolic deceleration time (DDT) of $161 \mathrm{msec}$, a peak diastolic velocity (PDV) of $45 \mathrm{~cm} / \mathrm{sec}$, and the presence of the systolic retrograde flow (SRF) were all consistent with the no-reflow phenomenon (Figure 4). Cardiac magnetic resonance imaging (MRI) was performed five days after the index event. It described extensive interstitial edema that affected the anterior, septal, and apical regions of the left ventricle. Areas of intramyocardial hemorrhage and microvascular obstruction (MVO) were also detected (Figure 5).

Beta-blocker treatment was initiated and up-titrated to $2.5 \mathrm{mg}$ bisoprolol b.i.d. Angiotensin converting enzyme inhibitor could not be associated due to low blood pressure values. The maximum dose of atorvastatin was adminis-

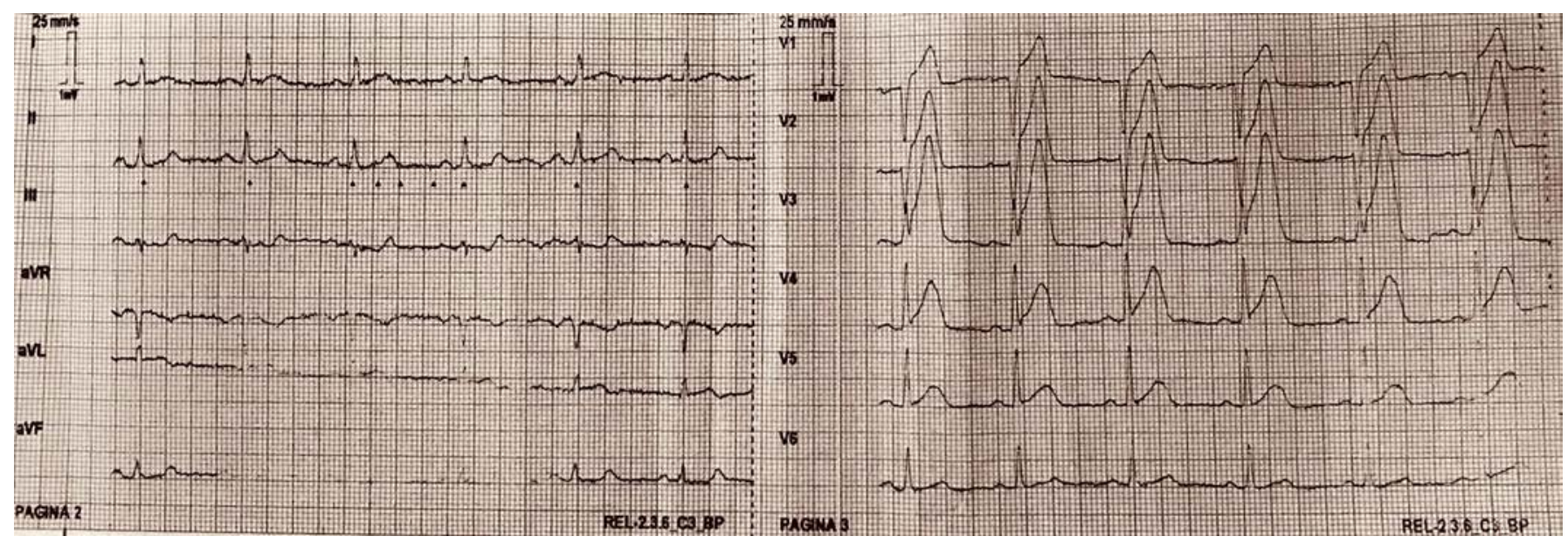

FIGURE 1. ECG recording at the time of admission: ST-segment elevation in the precordial leads 


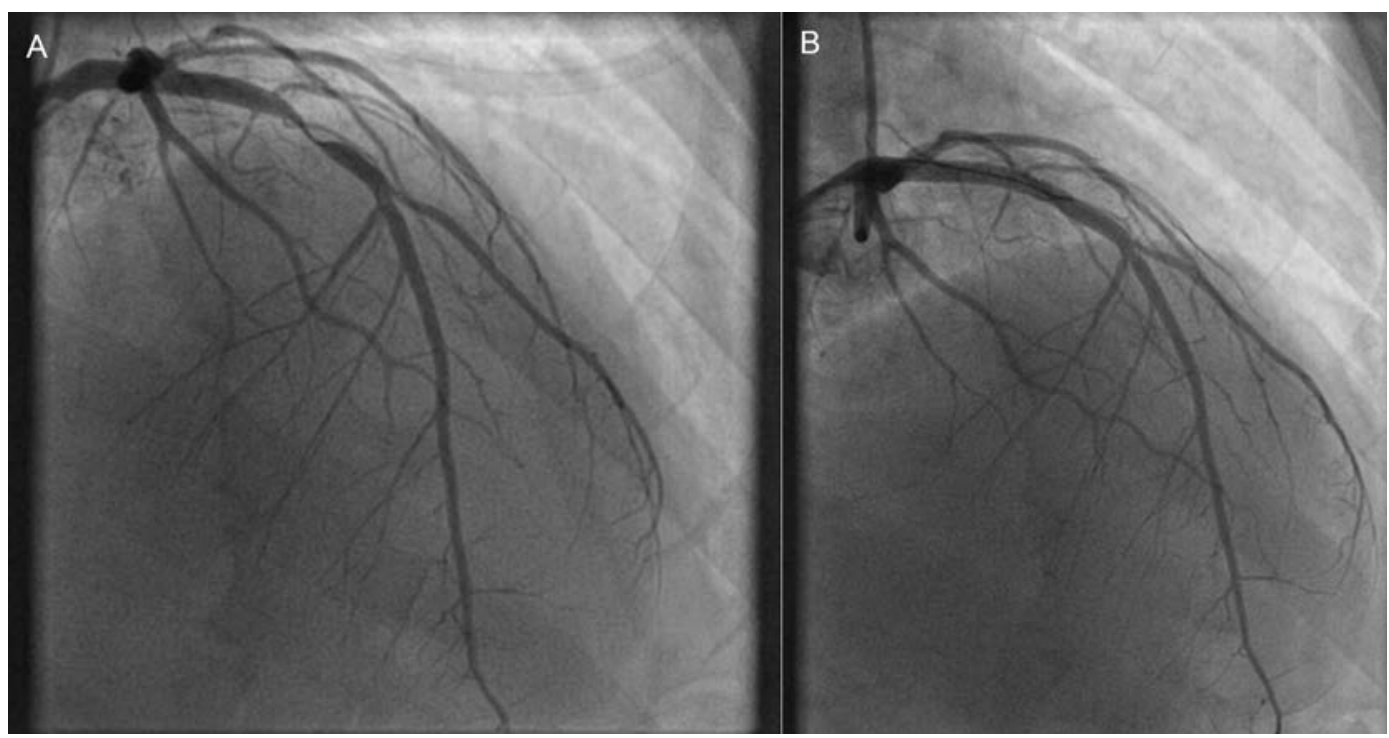

FIGURE 2. Right anterior oblique cranial angiographic views - Panel A: severe stenosis of the proximal left anterior descending artery; Panel B - final angiographic result (TIMI 3 flow, myocardial blush grade zero)

tered, and the patient was discharged on the seventh day of evolution.

The patient and the institution agreed with publication of this case.

\section{DISCUSSIONS}

The patient was an early presenter, with recently installed exertional angina. The ECG recording revealed the supra-acute phase of an acute myocardial infarction, in accordance with the above mentioned data. However, the angiographic result, as described by the MBG, was

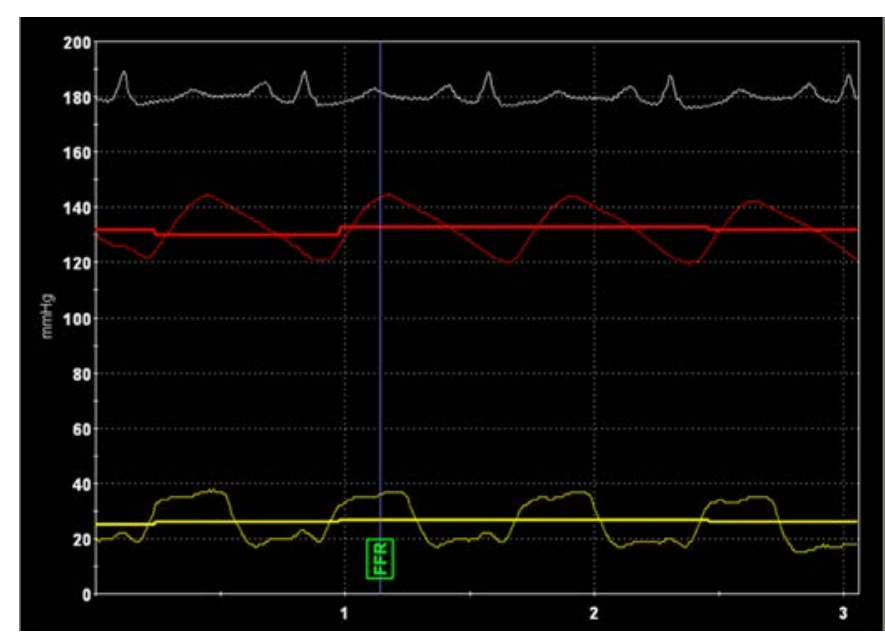

FIGURE 3. Coronary wedge pressure tracing: the mean pressure distal to the lesion is measured through the pressure-wire; a tall systolic wave, suggestive of distal embolism, can be observed (yellow trace) suboptimal, in spite of the TIMI 3 flow, suggesting the presence of MVO. ${ }^{9}$ Moreover, the absence of ST-segment resolution on the post-procedural ECG recording was also consistent with no-reflow phenomenon. Both STsegment resolution and MBG have prognostic utility after primary percutaneous coronary intervention (PCI). ${ }^{10}$ Both parameters predict mortality, left ventricular dysfunction, and clinical heart failure. ${ }^{11}$ The patient developed an apical left ventricular aneurysm, and his ejection fraction was depreciated.

Coronary blood flow interrogation by Doppler echocardiography emerged as a useful diagnostic tool for the noreflow phenomenon following acute STEMI. ${ }^{12}$ The increase in microvascular impedance secondary to microcirculatory obstruction is associated with a specific Doppler pattern. Among these parameters, DDT below 600 msec and the decrease of peak systolic velocity, up to the reversal of the systolic flow are the most powerful markers of microcirculatory dysfunction. ${ }^{13,14}$ In the case of this particular patient, DDT in the distal LAD was decreased to $161 \mathrm{msec}$, while PDV reached an increased value of $45 \mathrm{~cm} / \mathrm{sec}$. Together with the presence of SFR, these findings confirmed the presence of no-reflow phenomenon. ${ }^{12}$ Cardiac MRI confirmed the presence of MVO and especially its severity, as defined by the presence of interstitial edema and intramyocardial hemorrhage. Unfortunately, cardiac MRI, the gold standard for the evaluation of MVO, cannot be used prior to revascularization. As a result, it cannot influence the strategy for the early treatment of MVO. In this regard, coronary wedge pressure (CWP) appears to be a good surrogate parameter for the early detection of MVO. 


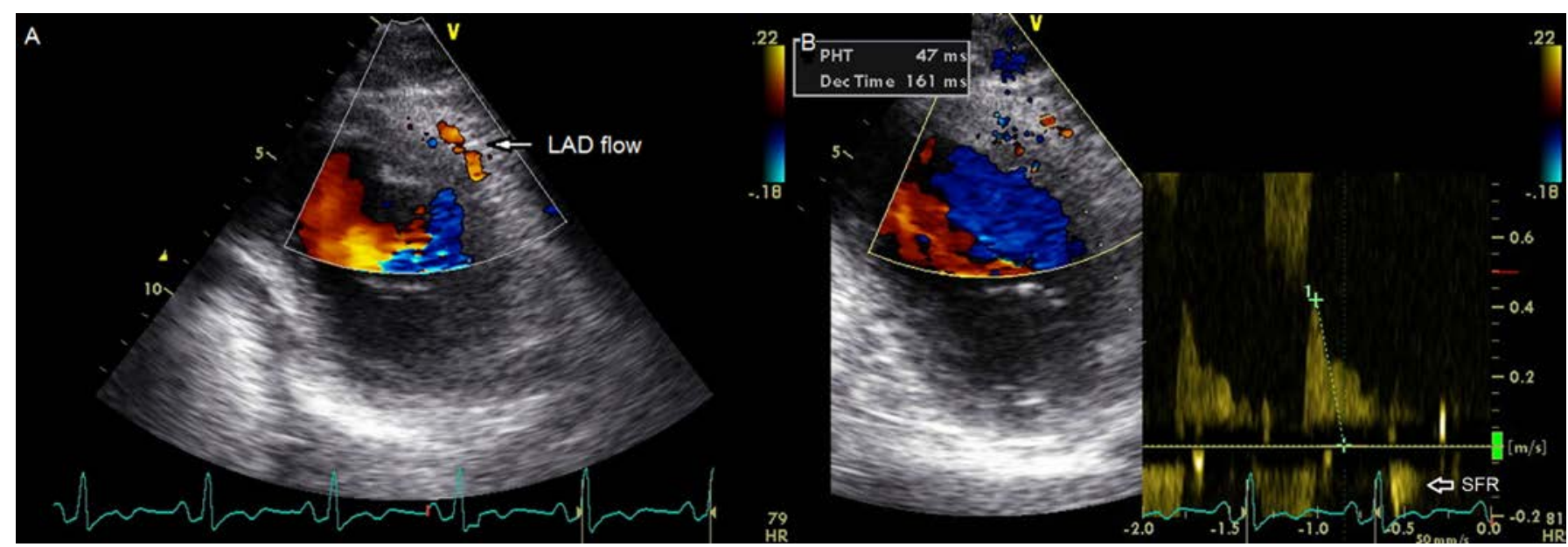

FIGURE 4. Left anterior descending artery flow assessed by Doppler echocardiography: the short diastolic deceleration time (161 msec) and the presence of systolic flow reversal (SFR) indicate the presence of no-reflow

Short time to reperfusion has been shown to be a powerful predictor of a good outcome in STEMI patients. However, total ischemic time is far from being the sole determinant of left ventricular remodeling or of an adverse prognosis. ${ }^{15}$

The platelet fresh component of the thrombus is unstable and prone to embolization into the distal microcirculation, causing MVO and interstitial inflammatory damage. ${ }^{16}$ The embolism that occurs several days be- fore the onset of symptoms was recently suggested by Kramer. ${ }^{15}$ Moreover, post-mortem studies on patients who died of non-revascularized acute coronary syndromes revealed that micro-emboli and inflammatory surroundings were more common in this subset of cases, clear proof of severe pre-procedural intramyocardial damage. ${ }^{16}$

Recently, Patel et al. have demonstrated that pressure at zero flow (Pzf) is the most sensitive invasive coronary

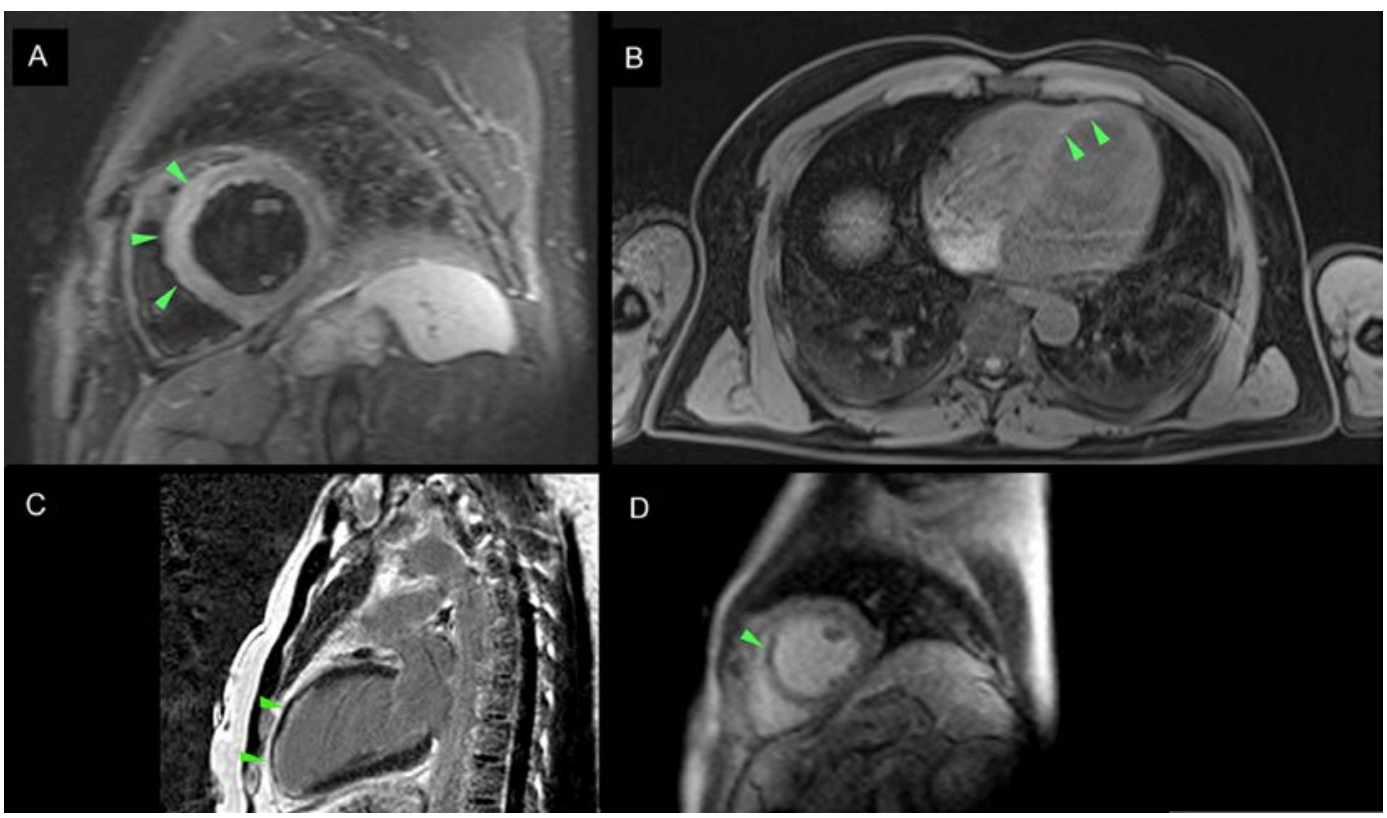

FIGURE 5. Cardiac magnetic resonance imaging - Panel A (short-axis view): edema of the interventricular septum; Panel B (transverse view): areas of intra-myocardial hemorrhage in the apical segment of the interventricular septum; Panel C (sagittal view): necrosis of the anterior wall of the left ventricle; Panel D (short-axis view): area of myocardial hypoenhancement in the interventricular septum, consistent with microvascular obstruction. 
physiology index currently available for the assessment of microcirculation at the time of primary PCI. ${ }^{17}$ In the setting of non-collateralized occlusions, CWP is similar to Pzf. Although both of them are pressure measurements at ceased coronary flow, Pzf is a theoretically derived hemodynamic parameter. On the other hand, CWP is the distal pressure in the occluded vessel after the occlusion is wired and reflects all the events which precede the revascularization procedure.

In the infarcted wall, MVO could impede coronary blood ejection into the venous circulation and could therefore cancel the benefit conferred in certain cases by coronary collateralization. This increases CWP by means of a large and tall systolic wave that defines the hemodynamic spectrum of $\mathrm{MVO} \cdot{ }^{18}$ On the other hand, CWP is also influenced by interstitial pressure as interstitial edema compresses capillaries and increases the intravascular pressure. ${ }^{19}$ The edema and inflammation surrounding the infarct zone are important determinants of left ventricular remodeling. ${ }^{20}$ A new coronary wave flow configuration occurs, with continuous elevation of the pressure line.

In the case of our patient, the high systolic wave in the CWP tracing suggested pre-procedural distal embolization and MVO. The therapeutic strategy, although in accordance with the current practice guideline, was only partly effective. Although the epicardial flow was restored, much of the microvasculature of the affected region remained obstructed. Cardiac MRI, the gold standard in the assessment of MVO, interstitial edema, left ventricular volumes, ejection fraction and infarct size, confirmed the presence of extensive interstitial edema, intramyocardial hemorrhage, and microvascular obstruction.

So, what could be done in order to improve the suboptimal long-term results in these patients? Intracoronary administration of glycoprotein IIb//IIIa inhibitors or thrombolytics could represent viable solutions in selected cases. ${ }^{21,22}$ In a previous study, we demonstrated a trend towards less MVO in patients who received distal intracoronary epitifibatide during primary PCI. ${ }^{23}$ This downstream delivery allowed for a high drug concentration to be established and avoided the use of the ineffective guidingcatheter administration.

But which is the most appropriate therapy? The two distal pressure wave patterns previously described could offer important information regarding the determinism of no-reflow in the setting of acute STEMI. A first scenario regards the pattern with a tall systolic wave that suggests embolism and MVO. It identifies the group of patients most likely to benefit from intracoronary glycoprotein IIb/ IIIa inhibitors or thrombolysis. In a second scenario, the continuous elevation of the pressure line is determined by the edema and the inflammation surrounding the affected area. These patients would probably benefit more from anti-inflammatory therapy. ${ }^{20}$

The current prophylactic and therapeutic measures for no-reflow and the consequent left ventricular remodeling in the setting of acute STEMI are either controversial or inadequate. Besides antiplatelet therapies and thrombolytics, several studies tested the benefit of thrombus aspiration or of distal protection devices. ${ }^{6,7}$ However, none of these have proved any benefit on MVO and left ventricular remodeling. Further investigation is needed in order to establish the effectiveness of each treatment and to determine the appropriate patient selection criteria.

\section{CONCLUSIONS}

In conclusion, there is more than meets the eye to coronary revascularization in the setting of an acute STEMI. Microvascular obstruction and interstitial edema are increasingly recognized as main players in the determinism of left ventricular remodeling. Moreover, MVO is not a temporally precisely located process. It starts before symptoms onset and continues during and after revascularization. The development of new treatment strategies will be a quest of the years to come. In this regard, the two different CWP patterns will differentiate the mechanism of MVO and will help select the most appropriate therapy.

\section{CONFLICT OF INTEREST}

Nothing to declare.

\section{REFERENCES}

1. Van't Hof W, Liem A, de Boer J, et al. Zwolle Myocardial Infarction Study Group. Clini-cal value of 12-lead electrocardiogram after successful reperfusion therapy for acute myo-cardial infarction. Lancet. 1997;350:615-619.

2. Resnic FS, Wainstein MW, Lee MK, et al. No-reflow is an independent predictor of death and myocardial infarction after percutaneous coronary intervention. Am Heart J. 2003;145:42-46. doi: 10.1067/mhj.2003.36.

3. Ito H, Tomooka T, Sakai N, et al. Lack of myocardial perfusion immediately after suc-cessful thrombolysis. A predictor of poor recovery of left ventricular function in anterior myocardial infarction. Circulation. 1992;85:1699-1705.

4. Ito H, Maruyama A, Iwakura K, et al. Clinical implications of the 'no-reflow' phenome-non: A predictor of complications and left ventricular remodeling in reperfused anterior wall myocardial infarction. Circulation. 1996;93:223-228.

5. Eitel I, Wöhrle J, Suenkel H, et al. Intracoronary Compared With Intravenous Bolus Abciximab Application During Primary Percutaneous Coronary Intervention in ST-Segment Elevation 
Myocardial Infarction: Cardiac Magnetic Resonance Substudy of the AIDA STEMI Trial. J Am Coll Cardiol. 2013;61:1447-1454. doi: 10.1016/j.jacc.2013.01.048.

6. Jolly SS, Cairns JA, Yusuf S, et al. Outcomes after thrombus aspiration for ST elevation myocardial infarction: 1-year follow-up of the prospective randomized TOTAL trial. Lancet. 2015;387:127-135. doi: 10.1016/S0140-6736(15)00448-1.

7. Stone GW, Webb J, Cox DA, et al. Distal microcirculatory protection during percutaneous coronary intervention in acute ST-segment elevation myocardial infarction: a randomized controlled trial. JAMA. 2005;293:1063-1072. doi: 10.1001/ jama.293.9.1063.

8. Kelbaek H, Terkelsen CJ, Helqvist S, et al. Randomized comparison of distal protection versus conventional treatment in primary percutaneous coronary intervention: the drug elution and distal protection in ST-elevation myocardial infarction (DEDICATION) trial.J Am Coll Cardiol. 2008;51:899905. doi: 10.1016/j.jacc.2007.10.047.

9. Soeda T, Higuma T, Abe N, et al. Morphological predictors for no reflow phenomenon af-ter primary percutaneous coronary intervention in patients with ST-segment elevation myocardial infarction caused by plaque rupture. Eur Heart J Cardiovasc Imaging. 2017;18:103-110. doi: 10.1093/ehjci/ jev341.

10. Brener SJ, Dizon JM, Mehran R, et al. Complementary prognostic utility of myocardial blush grade and ST-segment resolution after primary percutaneous coronary intervention: analysis from the HORIZONS-AMI trial. Am Heart J. 2013;166:676-683. doi: 10.1016/j.ahj.2013.07.025.

11. De Lemos JA, Braunwald E. ST Segment Resolution as a Tool for Assessing the Efficacy of Reperfusion Therapy. J Am Coll Cardiol. 2001;38:1283-1294.

12. Iwakura $\mathrm{K}$, Ito $\mathrm{H}$, Takiuchi $\mathrm{S}$, et al. Alternation in the coronary blood flow velocity pat-tern in patients with no reflow and reperfused acute myocardial infarction. Circulation. 1996;94:1269-1275.

13. Okamura A, Ito $\mathrm{H}$, Iwakura $\mathrm{K}$, et al. Usefulness of a new grading system based on coro-nary flow velocity pattern in predicting outcome in patients with acute myocardial infarction having percutaneous coronary intervention. Am J Cardiol. 2005;96:927-932. doi: 10.1016/j.amjcard.2005.05.049.

14. Iwakura $\mathrm{K}$, Ito $\mathrm{H}$, Kawano $\mathrm{S}$, et al. Assessing myocardial perfusion with the transthoracic Doppler technique in patients with reperfused anterior myocardial infarction: comparison with angiographic, enzymatic and electrocardiographic indices. Eur Heart J. 2004;25:1526-1533. doi: 10.1016/j. ehj.2004.06.029.

15. Kramer MC, Van der Wal AC, Koch KT, et al. Presence of older thrombus is an inde-pendent predictor of long-term mortality in patients with ST-elevation myocardial infarc-tion treated with thrombus aspiration during primary percutaneous coronary intervention. Circulation. 2008;118:1810-1816. doi: 10.1161/CIRCULATIONAHA.108.780734.

16. Schwartz RS, Burke A, Farb A, et al. Microemboli and Microvascular Obstruction in Acute Coronary Thrombosis and Sudden Coronary Death: Relation to Epicardial Plaque Histopathology. J Am Coll Cardiol. 2009;23:2167-2173. doi: 10.1016/j.jacc.2009.07.042.

17. Patel N, Petraco R, Dall'Armellina E, et al. Zero-Flow Pressure Measured Immediately After Primary Percutaneous Coronary Intervention for ST-Segment Elevation Myocardial Infarction Provides the Best Invasive Index for Predicting the Extent of Myocardial In-farction at 6 Months: An OxAMI Study (Oxford Acute Myocardial Infarction). J Am Coll Cardiol Intev. 2015;8:1410-1421. doi: 10.1016/j.jcin.2015.04.029.

18. Sezer M, Umman B, Okcular I, NisanciY, Umman S. Relationship between microvascular resistance and perfusion in patients with reperfused acute myocardial infarction. J Interv Cardiol. 2007;20:340-350. doi: 10.1111/j.1540-8183.2007.00274.x.

19. Echavarría-Pinto M, Serruys PW, Garcia-Garcia HM, et al. Use of intracoronary physiol-ogy indices in acute coronary syndromes. Interventional Cardiology. 2015;7:5,483-495.

20. Westman PC, Lipinski MJ, Luger D, et al. Inflammation as a Driver of Adverse Left Ven-tricular Remodeling After $\underline{\text { Acute }}$ Myocardial Infarction. J Am Coll Cardiol. 2016;67:2050-2060. doi: 10.1016/j.jacc.2016.01.073.

21. Qin T, Xie L and Chen MH. Meta-analysis of randomized controlled trials on the efficacy and safety of intracoronary administration of tirofiban for no-reflow phenomenon. BMC Cardiovasc Disord. 2013;13:68. doi: 10.1186/1471-2261-13-68.

22. Sezer M. Oflaz H, Gören T, et al. Intracoronary Streptokinase $\underline{\text { after }}$ Primary Percutaneous Coronary Intervention. N Engl J Med. 2007; 356:1823-1834. doi: 10.1056/NEJMoa054374.

23. Iancu A, Ober C, Bondor CI, Cadiş H. Microvascular effect of intracoronary eptifibatide in acute myocardial infarction. Cardiology. 2012;123:46-53. doi: 10.1159/000341197. 